\title{
Reply to "Could a mannequin simplify rhinopharyngeal swab collection in COVID 19 patients?"
}

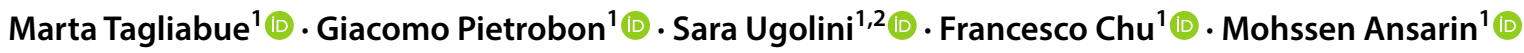

Received: 27 June 2020 / Accepted: 2 July 2020 / Published online: 18 July 2020

c) Springer-Verlag GmbH Germany, part of Springer Nature 2020

Keywords COVID-19 · Nasopharyngeal swab · Endonasal endoscopy

Dear Editor,

We welcome the opportunity to comment on the recent paper by Boscolo Nata et al. because it allows some important elucidations [1].

First of all, sampling of the nasopharynx under endoscopic endonasal control is meant only for the training of the healthcare personnel: it is performed on healthy volunteers and aimed at providing a direct and clear visualization of both the correct technique and the possible variants of endonasal anatomy. For this reason, there are neither risks of exposure nor increased costs for sterilization. Of note, discomfort for the volunteer is negligible, because topical anesthetic and vasoconstrictor are applied ahead of the procedure, which is then performed by an experienced otolaryngologist. Endoscopic-assisted sampling on patients should be reserved only to extraordinary cases, where anatomical variants are anticipated on the basis of medical history, as recently suggested by De Virgilio et al. [2].

Although the viral load of different endonasal sites has not been analyzed so far, so that sampling of the nasal fossa or middle meatus could potentially provide valuable results as well, the current international guidelines recommend that the nasopharynx is swabbed for its demonstrated higher sensitivity [3].

This reply refers to the comment available online at https://doi. org/10.1007/s00405-020-06113-5.

Giacomo Pietrobon

giacomo.pietrobon@gmail.com

1 Division of Otolaryngology and Head and Neck Surgery, European Institute of Oncology IRCCS, Via Ripamonti 435, Milan, Italy

2 Department of Otorhinolaryngology, IRCCS Fondazione Policlinico San Matteo, University of Pavia, Pavia, Italy
Nasopharyngeal sampling is not an ordinary procedure and yet, at the beginning of the pandemic, training of the healthcare personnel had to be as fast and effective as possible because of the daily-increasing requirements. In our opinion, engagement of ENT specialists and endoscopes for visual aid represented the best option for such a training. A real-life endoscopic demonstration is favored by the possibility of showing not only the correct approach to the nasopharynx, but also the potential mistakes in sampling and the feasible maneuvers to overcome any endonasal obstacles and the subject's involuntary movements. In our experience, it takes a very short time for the attendants to become familiar with the procedure and the hints of nasal anatomy. Once this training is complete, otolaryngologists may supervise the first 5-10 samplings on patients in order to provide further help or clarifications while minimizing self-exposure.

In our opinion, the use of a mannequin comes with several shortcomings. First, such a device, especially if sectioned on the midline, is unlikely to be available in ordinary times, let alone during a pandemic in a peripheral primarycare hospital. Proof is that even the proposing Authors were not able provide a picture of this model. Second, the mannequin's anatomy is standardized, likely with a straight nasal septum and eutrophic turbinates, and does not reproduce the high variability of human beings, so that training on it would be misleading. Third, a mannequin does not allow for visual feedback of the whole procedure and its possible mistakes and of the subject's reactions (e.g., sneeze when incorrectly swabbing the middle meatus).

Healthcare personnel dedicated to nasopharyngeal swab collection should not be trained in the simple robotic act of sampling, but should be provided with some knowledge of nasal and nasopharyngeal anatomy as well, in order to increase the accuracy of the swab and to avoid any possible error. Nowadays, ENT departments are widely equipped with endoscopes, both rigid and flexible, and, in the time of 
COVID19, otolaryngologists were often set aside their daily duties. Lecturing on nasal anatomy is one of their chances to participate actively in this pandemic.

In conclusion, we thank the Editor for the opportunity to explicit some interesting points of debate. Nevertheless, in light of the abovementioned limits, we continue to prefer personalized healthcare personnel training aided by endoscopic endonasal view [4].

Funding This work was partially supported by the Italian Ministry of Health with Ricerca Corrente and $5 \times 1000$ funds.

\section{Compliance with ethical standards}

Conflict of interest Nothing to declare.

Ethical approval Not applicable.

Ethical standards Not applicable.

Informed consent Not applicable.

\section{References}

1. Francesca BN, Barbara P, Emanuele F et al (2020) Could a mannequin simplify rhinopharyngeal swab collection in COVID 19 patients? Eur Arch Otorhinolaryngol 3:96. https://doi.org/10.1007/ s00405-020-06113-5

2. De Virgilio A, Pellini R, Mercante G et al (2020) Who should perform the rhinopharyngeal swab in COVID-19 positive patients? Head Neck 42(6):1250-1251. https://doi.org/10.1002/hed.26194

3. Centers for Disease Control and Prevention, Interim Guidelines for Collecting, Handling, and Testing Clinical Specimens from Persons for Coronavirus Disease 2019 (COVID-19), https://www. cdc.gov/coronavirus/2019-ncov/lab/guidelines-clinical-speci mens.html. Accessed 15 June 2020

4. Tagliabue M, Pietrobon G, Ugolini S et al (2020) Nasopharyngeal swabs during SARS-CoV-2 pandemic: a role for the otolaryngologist. Eur Arch Otorhinolaryngol 277(7):2155-2157. https://doi. org/10.1007/s00405-020-06027-2

Publisher's Note Springer Nature remains neutral with regard to jurisdictional claims in published maps and institutional affiliations. 